\title{
Properties of a hypothetical cold pulsar wind in LS 5039 (Corrigendum)
}

\author{
V. Bosch-Ramon
}

\author{
Departament de Física Quàntica i Astrofísica, Institut de Ciències del Cosmos (ICC), Universitat de Barcelona (IEEC-UB), \\ Martí i Franquès 1, 08028 Barcelona, Spain \\ e-mail: vbosch@fqa.ub.edu
}

A\&A 645, A86 (2021), https://doi .org/10.1051/0004-6361/202039666

Key words. gamma rays: stars - radiation mechanisms: non-thermal - stars: winds, outflows - stars: individual: LS 5039 errata, addenda

Unfortunately, Eq. (10) in Bosch-Ramon (2021) was incorrectly written. However, the calculations presented in the paper were performed with the correct (approximated) expression for that angle, which is:

$\tau \approx\left(1+\eta^{1 / 2}\right) \frac{\omega_{\mathrm{orb}} R_{\mathrm{orb}}}{\left|\boldsymbol{v}_{\mathrm{w}}\right|}$ where $\left|\boldsymbol{v}_{\mathrm{w}}\right|$ is the modulus of the stellar wind velocity vector in the orbit-rotating frame, $\omega_{\text {orb }}$ the pulsar orbital angular velocity, $R_{\text {orb }}$ the orbital separation distance, and $\eta$ the pulsar-to-star wind momentum rate ratio.

\section{(1) References}

Bosch-Ramon, V. 2021, A\&A, 645, A86 